\title{
3D DATA PRODUCTS AND WEB-GIS FOR MARS ROVER MISSIONS FOR SEAMLESS VISUALISATION FROM ORBIT TO GROUND-LEVEL
}

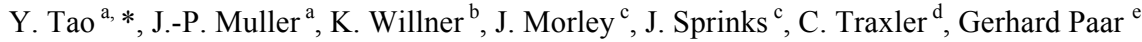 \\ ${ }^{a}$ Imaging Group, Mullard Space Science Laboratory, University College London, Holmbury St Mary, Dorking, Surrey,RH56NT, UK \\ (yu.tao,j.muller)@ucl.ac.uk \\ ${ }^{\mathrm{a}}$ Department of Geodesy and Geoinformation Science, Planetary Geodesy, Technische Universität Berlin, 10623 Berlin, Germany \\ konrad.willner@tu-berlin.de \\ ${ }^{c}$ Nottingham Geospatial Institude, Triumph Road, University of Nottingham, Nottingham, NG72TU, UK \\ (jeremy.morley, psxjs6)@nottingham.ac.uk \\ ${ }^{\mathrm{d}}$ VRVis Zentrum für Virtual Reality und Visualisierung Forschungs-GmbH, Donau-City-Strasse, Wien, Austria - traxler@vrvis.at \\ 'JOANNEUM RESEARCH Forschungsgesellschaft mbH, Steyrergasse 178010 Graz, Austria - gerhard.paar@joanneum.at
}

\section{Commission IV, WG IV/8}

KEY WORDS: HRSC, CTX, HiRISE, MER, MSL, ORI, DTM, co-registration, wide baseline stereo reconstruction, ground-to-orbit fusion, web-GIS, 3D visualisation

\section{ABSTRACT:}

This paper presents a wide range of research and processing results in the area of multi-resolution orbital data co-registration, multiresolution ground 3D reconstruction, and orbit-to-ground data fusion, achieved within the EU-FP7 PRoVisG and PRoViDE project. We focus on three NASA rover missions, MER-A, MER-B, and MSL, to provide examples of automated methods for producing coregistered, multi-resolution 3D products. We highlight the mis-registration discovered between current HiRISE to HRSC datasets, CTX to HRSC and HiRISE to CTX co- results, wide baseline stereo reconstruction results of rover imagery, ground-to-orbit coregistration, i.e. reconstructed wide baseline ground ORI and HiRISE ORI co-registration, and extensive exploitation of the coregistered datasets in visualisation and interactive web-GIS.

\footnotetext{
* Corresponding author.
} 


\section{INTRODUCTION}

Planetary images and 3D data derived from stereo images therein collected from past and on-going missions usually focus on different specific requirements and are mostly treated separately. However, to analyse an area of interest properly or to do any extensive geological classification and interpretation requires $3 \mathrm{D}$ information at various level of details. This report will describe a wide range of developments and the processing results achieved within the EU-FP7-PRoVisG (Planetary Robotic Vision Ground Processing) project (http://provisg.eu), which was carried out from 2008 to 2012, and the 3 year EUFP7-PRoViDE (Planetary Robotic Vision Data Exploitation) project (http://provide-space.eu) that started in 2013 to collect all the multi-view imaging data from ground-level robotic and orbital sensors covering three Mars rover missions, i.e. MER-A, MER-B (Mars Exploration Rover), and MSL (Mars Science Laboratory), and process them into a coherent set of coregistered $3 \mathrm{D}$ imaging products [1]. Bringing them into the most accurate possible co-registration allows their exploration through interactive real-time visualisation tools and Web GIS.

This work commenced with the automated co-registration of common features in orbital datasets, i.e. the NASA Mars Reconnaissance Orbiter HiRISE (High Resolution Image Science Experiment) to CTX (Context Camera), and thence to the ESA Mars Express HRSC (High Resolution Stereo Camera), over 3 rover landing and exploration sites: MER-A/-B and MSL. In order to resolve the mis-registration issue reported in [2] between HiRISE and HRSC, we processed CTX images to derive orthorectified Images (ORIs at $6 \mathrm{~m} /$ pixel) and Digital Terrain Models (DTMs) at (18 m/grid point) using the publicly available NASA Ames Stereo Pipeline (ASP) [3]. These CTX ORIs and DTMs were then co-registered to the HRSC ORI $(12.5 \mathrm{~m} / \mathrm{pixel}) / \mathrm{DTM}$ (75m/pixel) which are themselves coregistered with the Mars Orbiter Laser Altimeter (MOLA) data. The HiRISE ORI $(0.25 \mathrm{~m} /$ pixel $) / \mathrm{DTMs}(1 \mathrm{~m} / \mathrm{pixel})$ were then separately co-registered to the CTX image to form a multiresolution cascade [4] of co-registered ORIs and DTMs. In the second stage, we generate multi-resolution ground level 3D reconstructed products (ORIs and DTMs) from different instruments onboard the rovers, e.g. MSL MastCam and NavCam, providing angular resolution from $0.074 \mathrm{mrad} /$ pixel to $0.82 \mathrm{mrad} /$ pixel respectively. Wide baseline stereo ORI/DTM were then reconstructed from pairs of stereo images, i.e. NavCam images, via region growing/ALSC [5] based on intrastereo and Bundle adjustment for inter-stereo processing. Images from non-stereo cameras (e.g. MSL MAHLI) are then co-registered and ortho-rectified based on NavCam ORI/DTMs. For close range, we are able to produce better than $10 \mathrm{~mm} / \mathrm{pixel}$ resolution ORI/DTMs (e.g. 10mm/pixel NavCam, $3 \mathrm{~mm} /$ pixel MastCam-34, and $1 \mathrm{~mm} /$ pixel MastCam-100) at this stage. Subsequently, the reconstructed wide baseline ground ORI/DTMs were co-registered with HiRISE ORI/DTM using corresponding features found from edges and corners. These correspondences are then used to provide an updated rover transect where the actual rover position can be assessed against common features visible in the rover and orbital images.

These digitised multi-resolution 3D ground-level products enable the "virtual geologist" to perform close-up visual analysis of key features (e.g. sedimentary layers) and will allow future geometrical measurements (e.g. distance, dip and slope) with different level of details in a common global context. The 3D products have also been inserted into our interactive WebGIS system, i.e. PRoGIS (http://progisweb.eu) being developed in PRoVisG and upgraded within the scope of PRoViDE by the University of Nottingham in collaboration with UCL-MSSL, to serve the educational, publicity and scientific objectives of our research [6]. In the future, the SPICE kernels will be updated to ensure that there is consistency with all datasets from orbit to ground level.

\section{METHODS}

\subsection{Automated tie-point based co-registration of HiRISE, CTX and HRSC}

The availability of HiRISE images has brought the rover localisation work to a new level of precision. However, when taking a closer look at the HiRISE ORI/DTM (from the NASA HiRISE site) and comparing it to a HRSC ORI/DTM, we found that they are not precisely co-registered to each other (see Fig.1). There are mis-registrations of about $100 \mathrm{~m}$ for the MER-A HiRISE to HRSC, $100 \mathrm{~m}$ to $150 \mathrm{~m}$ for the MERB site for HiRISE to HRSC, and from $100 \mathrm{~m}$ to $200 \mathrm{~m}$ for the MSL site for HiRISE to HRSC, according to manually selected control points on obvious features, e.g. craters. After selecting homologous tiepoints and applying a second order transformation, the misregistration can be reduced to pixel level [2]. However, this had the unintended consequence that the rover traverses, which were corrected with respect to the landing site reference coordinates using incremental bundle adjustment (IBA) based on matching features from look-ahead/behind optical navigation [7], no longer fitted visible features on the HiRISE map. It became obvious when trying to place rover traverses (MER and MSL) in context that such traverses did not match with known landmarks visible in the orbital images. After experimenting with different approaches it appears that the use of equirectangular projection and poor co-registration of HiRISE to HRSC meant that a different approach had to be undertaken. Consequently, we decided to take CTX ORI as a resolution bridge, applying an automated tie-pointing method to coregister the HiRISE to the CTX and subsequently the CTX to the HRSC ORI datasets. This allows the co-registration accuracy to be improved from pixel level to sub-pixel level. We processed CTX stereo pairs (B18 016677 1653 XN $14 \mathrm{~S} 184 \mathrm{~W}$ and G01 0185231653 XI $14 \overline{\mathrm{S}} 184 \mathrm{~W}$ for MER-A, B22_018134_1779_X XN_02S005W and G01_018490_1779

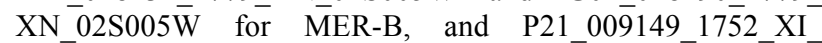
04S222W and P21_009294_1752_XI_04S222W for MSL) to derive ORI and DTM of $6 \mathrm{~m} / \mathrm{pixel}$ spatial resolution, using the NASA ASP software. CTX acts as a bridge between the $0.25 \mathrm{~m}$ MRO HiRISE and 12.5-25m HRSC ORIs. We use the HiRISE ORI/DTM and ORI/DTM mosaic produced by our US collaborators at the Mapping and GIS Laboratory, Ohio State University (OSU), for MER-A and MER-B. They reported their rigorous photogrammetric model for HiRISE and coarse-to-fine hierarchical matching approach in [8]. The HiRISE images are processed from stereo pairs (PSP_001513_1655 and PSP 0017771650 for MER-A, PSP 009141 1780 and PSP_001414_1780, ESP_011765 1780, ESP 012820 1780 and ESP_021536_1780, PSP_004289_1780, PSP_010341_1775 and PSP $010486 \_1775$ for MER-B Mosaic) and the products have a spatial resolution of $0.25 \mathrm{~m} /$ pixel for MER-A HiRISE ORI, $0.33 \mathrm{~m} / \mathrm{pixel}$ for MER-B HiRISE ORI Mosaic, and $1 \mathrm{~m} /$ pixel for the DTMs. For MSL, we use the HiRISE ORI/DTM produced by the U.S. Geological (USGS) Astrology team using ISIS and SOCET SET software [9]. The MSL HiRISE ORI and DTM have the spatial resolution of $1 \mathrm{~m} / \mathrm{pixel}$. 


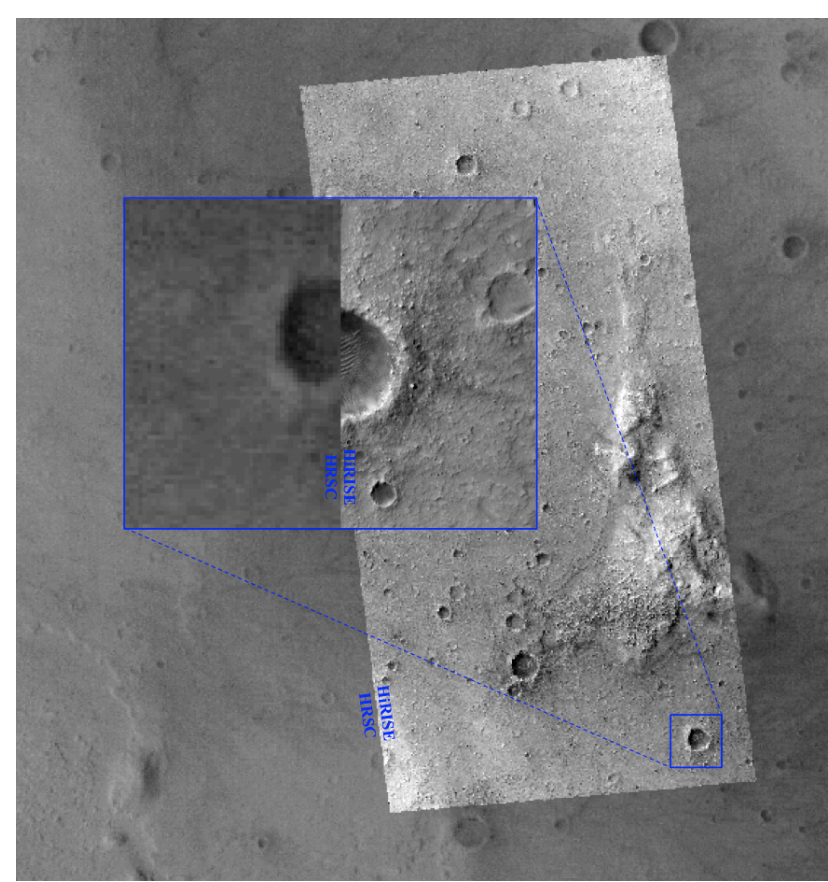

Figure 1. Example swipe view showing mis-registration between MER-A HiRISE ORI and HRSC ORI

We projected the CTX and HiRISE ORI/DTM from Mars 2000 aerographic coordinate system into the Sinusoidal projection system with the same central meridian value, which is originally used in HRSC datasets, i.e. $176^{\circ} \mathrm{E}$ for MER-A, $354^{\circ} \mathrm{E}$ for MER$\mathrm{B}$, and $138^{\circ} \mathrm{E}$ for MSL. The co-registration method applied is illustrated schematically in Fig.2.
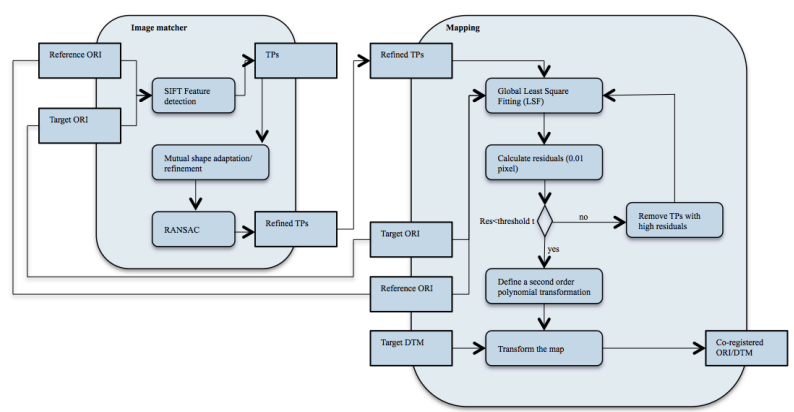

Figure 2 Flow diagram of HiRISE-CTX-HRSC co-registration

We start with detecting feature points using the Scale Invariant Feature Transform (SIFT), which is widely used in image matching. However, this general feature based matching method assumes that the image features detected independently on each image are always correct. The repeatability of the detection is deteriorated when a significant distortion is involved in a matching image. Slight mismatch can bring a large impact on the global transformation. Therefore, we developed a Mutual Shape Refinement (MSR) algorithm that combines the scale and affine invariant feature detector with Adaptive Least Square Correlation (ALSC) to refine an approximated matching result and obtain more accurate Tie-Points (TPs). See Fig.3 for results. The algorithm of mutual shape refinement consists of i) Detection of a scale invariant feature and its scale ii) Iteratively update a circular scale invariant region to an elliptical region using a second moment matrix iii) Initial normalisation using the result from ii.

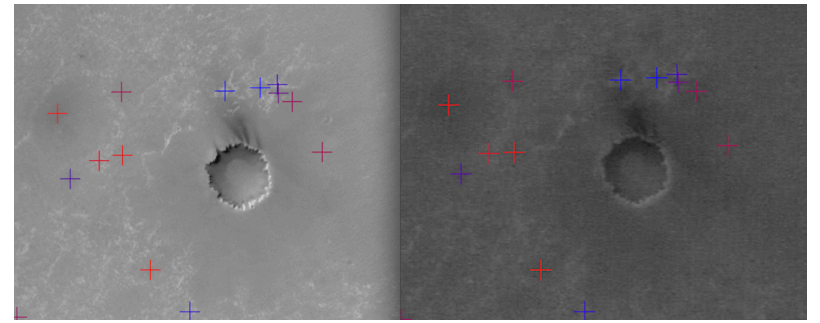

Figure 3 Example of detected TPs (showing from red to blue with increasing similarity value $0-1$ ) for MER-B CTX ORI(left) and HRSC ORI (right) at Victoria Crater

iv) Refine the result using forward and backward ALSC on both images v) Go back to iv until it reaches a termination point (optional) vi) Go back to ii until it reaches a termination point (optional but not necessary).

The next step is to define a $2^{\text {nd }}$ order polynomial transformation from the refined TPs. The transformation is decided according to the Least Squares Fitting (LSF) algorithm and adjustment of local TPs for better fitting of the global TPs using TIN interpolation. The residuals are calculated iteratively and outliers are removed until they reach a threshold. The final transformation for the ORIs is then applied to the DTMs. The co-registered datasets then act as a reference base image for the co-registration of the reconstructed wide baseline ground ORI, which will be described in the following section.

\subsection{Multi-resolution Wide Baseline Ground Reconstruction}

Due to very large scale and viewpoint differences, it is not easy to link a single ground image to an orbital image. Even if it were possible, the result would not be useful when the coverage of the ground view is very small in the orbital view. Therefore a wide baseline reconstruction is essential to link ground products with orbital datasets. We developed a processing chain based on tiled region growing and ALSC for intra-stereo pairs, and then back projecting $3 \mathrm{D}$ point clouds onto inter-stereo pairs and applying Bundle Adjustment (BA) to build an image network.

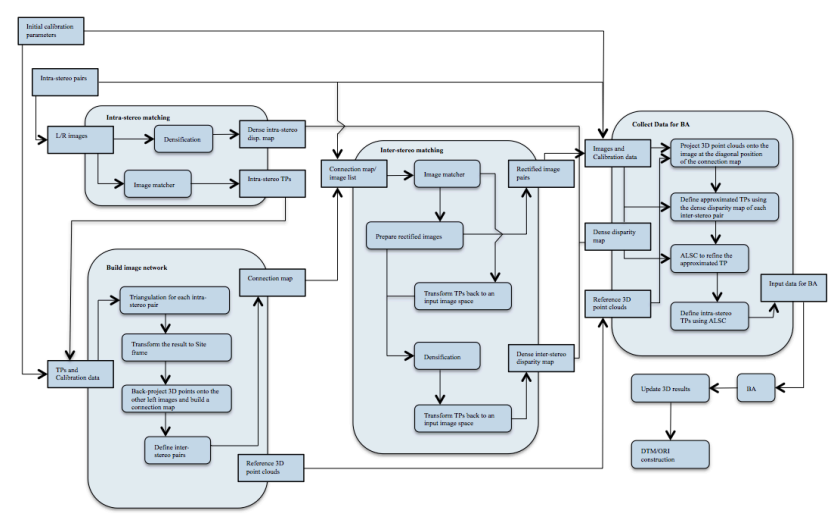

Figure 4. Flow diagram of implemented wide baseline stereo reconstruction processing chain

Partial or full stereo panoramas were normally taken at the end of a drive. Craters, cliffs, and hills are the most important features from these ground maps. Wide baseline reconstructions of such areas could be more effectively co-registered with orbital ORI/DTMs. For MER-B, the data collected around, e.g. Eagle Crater (Sol 1 to Sol 65), Endurance Crater (Sol 95 to Sol 318), Victoria Crater (Sol 950 to Sol 1683), Santa-Maria Crater (Sol 2450 to Sol 2542), contains large numbers of wide baseline panoramas with significant structural feature that are viewable from HiRISE ORI/DTM. For MER-A, a similar situation 
appears around the Columbia Hills (Sol 155 to Sol 581), and HomePlate (Sol 745 to Sol 2180). For MSL, there are many wide baseline panoramas with rich features appearing in Shaler (Sol 120 and Sol 121), Yellowknife Bay (Sol 124 to Sol 299), Gillespie Lake (Sol 130 to Sol 133) and Cumberland (Sol 274 to Sol 295), of already released data. We initially sought for serendipitous stereo pairs from the fulcra file derived from the raw Navigation and Ancillary Information Facility (NAIF) SPICE kernels. In this stage, computation of the approximated fulcra assumes a simple surface of the rover surroundings. A simple SIFT/SURF based feature detection and matching process was employed where necessary to define potential wide baseline panoramas. The fulcra file are subsequently updated applying the derived local DTMs or HiRISE DTMs after BA and ground-to-orbit co-registration for further refinement of these field of view footprints.

The wide baseline reconstruction generally can be addressed with 3 cases: 1) Panning motion of the same sensor at a fixed rover position; 2) Panning motion of different sensors, i.e. NavCam and PanCam for MER, MastCam and NavCam for MSL, at a fixed position or a general motion of the same/different sensors, i.e. different rover positions; 3) crosssite stereo pairs. We only focus on cases 1 and 3, because largescale structural information is more useful for the fusion with HiRISE ORI compared with detailed local structure from MER PanCam and MSL MastCam reconstructions.

MER and MSL camera calibration data is defined in the rover coordinate system whilst the reconstruction result, i.e. XYZ data uses the Site coordinate system. A transformation between a rover frame and its site frame can be defined using 7 parameters in the PDS header, i.e., 3 offset and 4 quaternion parameters.

We initially use the CAHVOR camera coefficients recorded in the PDS header for intra-stereo reconstruction and inter-stereo network building. For intra-stereo reconstruction, a SIFT/SURF feature based matching is used to obtain a list of sparse TPs followed by a mutual shape refinement. TPs are used to rectify the left and right images into epipolar geometry. In the densification/stereo-matching step, a region growing/ALSC based approach is used to produce dense disparity maps. After triangulation of each intra-stereo pair, BA is used to correct the inter-stereo image network and update the extrinsic calibration data. For close range $(<5 \mathrm{~m})$, we are able to produce better than $10 \mathrm{~mm} /$ pixel resolution ORI/DTMs.

We build a wide baseline image network through 5 steps: 1) 3D triangulation from each intra-stereo pair; 2) Back projection of point clouds to each left image; 3) Define inter-stereo pairs; 4) Link TPs between inter-stereo pairs; 5) Link TPs between intrastereo pairs.

Since MER PanCam and MSL MastCam have a higher resolution for far range objects but a narrower FOV, NavCam are more suitable for wide baseline stereo reconstruction and ground-to-orbit co-registration. Although stereo reconstructions from MER PanCam or other 2D images from science cameras, e.g. MastCam, MAHLI, ChemCam, enable very detailed analysis of particular features of interest, these can be reconstructed to $3 \mathrm{D}$ and co-registered with global context taking NavCam 3D products as a bridge [10]. An end result is a set of full-resolution NavCam 3D point clouds $(0.82 \mathrm{mrad} / \mathrm{pixel})$ represented as ORI/DTM which are in a common georeferenced framework along with higher resolution PanCam or MastCam 3D point clouds (PanCam: $0.28 \mathrm{mrad} /$ pixel, M-34: 0.22 $\mathrm{mrad} / \mathrm{pixel}$ and M-100: $0.074 \mathrm{mrad} / \mathrm{pixel}$ ) wherever PanCam or MastCam34/100 can be employed to generate much denser and finer 3D points and image texture.

\subsection{Ground-to-orbit Co-registration}

The NASA MER project has employed Incremental Bundle Adjustment (co-registration of features looking ahead and the same features looking behind) as well as manual co-registration of rover imagery with rover images for rover localisation. A comparison of both methods performed is reported in [11]. However, close inspection of the IBA transect with HiRISE ORIs showed large discrepancies and the aforementioned intercomparison required that brute force methods needed to be employed to line up both transects in the first place. Here, this step is eliminated by ensuring the maximum accuracy possible between the rover images and their tracks visible in the orbital images. To relate close range views with global context views is challenging but critical in ground-to-orbit data fusion and rover localisation. Possible solutions include: 1) Fusing data in the 2D domain (multi-resolution/view image matching); 2) Fusing data in the 3D domain (3D data co-registration); 3) Fusing 3D data through 2D images (multi-dimensional and multi-resolution matching). In this paper, we describe a combined feature and area based registration method, which has been employed in alignment of wide baseline NavCam ORI mosaics and HiRISE ORI(s).

Since the spatial resolution varies in the local view, to avoid inaccurate depth estimation from a NavCam stereo pair, the depth range is limited to $-20 \mathrm{~m}$ to $40 \mathrm{~m}$. The best reconstruction distance for producing less-distorted NavCam ORI would be less than $15 \mathrm{~m}$. In practice, we limit the cut-off distance from $10 \mathrm{~m}$ to $25 \mathrm{~m}$ to pick up any extractable feature that could be matched with the HiRISE ORI. Features further than $25 \mathrm{~m}$ are not useful as the spatial quality and resolution is too low.

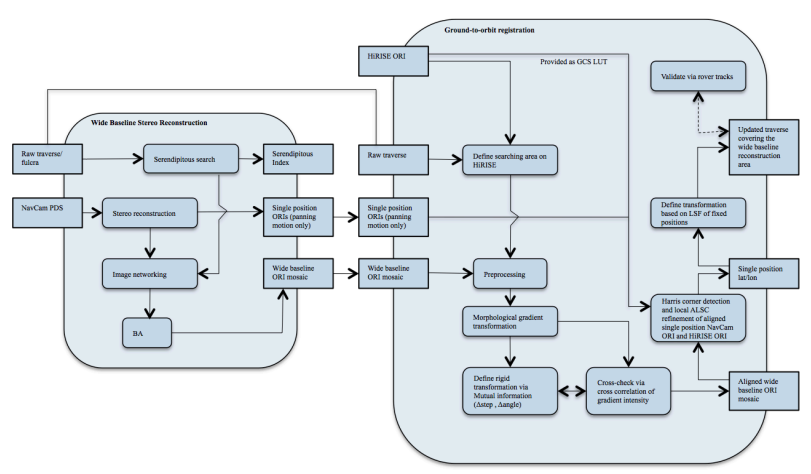

Figure 5. Flow diagram of implemented ground-to-orbit fusion processing chain

We initially use the information from raw traverse data in the rover reference system and landing site aerographic coordinates in a global reference system to define a search area on the corresponding HiRISE ORI (see Fig. 5). The search area does not necessarily need to be accurately set as its purpose is to try to maximize the automation of our processing chain. Alternatively, one can define an approximate area containing the reconstructed wide baseline ORI manually. The preprocessing includes a series of bilateral and Gaussian filtering kernels and interpolation processes, in order to smooth out noise generated in the NavCam 3D reconstruction and orthorectification, and local textures that do not appear in the HiRISE ORI. In the meantime, the structural texture that is likely to appear in the HiRISE ORI remains and is initially enhanced in the pre-processing stage. After pre-processing, the NavCam ORI mosaic and HiRISE ORI (subset) are scaled into the same spatial resolution, i.e. $0.05 \mathrm{~m} / \mathrm{pixel}$ or $0.1 \mathrm{~m} / \mathrm{pixel}$ in general, in order to save calculation time in matching, taking 
advantage of known geometrical constraints, i.e. intrinsic calibration parameters of NavCam. The next step is to apply a morphological gradient transformation to further enhance the structural features in both the NavCam ORI mosaic and HiRISE ORI. The processed NavCam ORI mosaics and HiRISE ORI are then co-registered using the normalised Mutual Information with defined pixel precision and angular step size. We use the normalised form of mutual information in order to eliminate the effects of unreliable joint histogram yield by local texture, i.e. as the NavCam ORI mosaic moves away from the optimal coregistration point that is detected by enhanced structural texture, mutual information can still increase if the increase of marginal entropies exceeds the decrease of the joint entropy between them. Radiometric differences are handled by a gradient descent approach to the least squares formulation. A rigid transformation, i.e. only translation and rotation are corrected, will be determined in this step. There is an optional cross checking step to look for local cross correlation of the derived gradient intensity. At this stage, the single position ORI that contains only panning motion of the cameras is located on the HiRISE ORI based on the aligned wide baseline ORI that contains this single position ORI. "Harris" corners are detected with the two aligned ORIs followed by a local ALSC refinement. The final transformation of the inquired position is determined based on the LSF of the local ALSC refinement result. The traverse covering the reconstructed wide baseline area is corrected after all positions have been refined. The matching results depend greatly on two factors: 1) the wide baseline reconstruction covers a large enough area. 2) the area must have rich structural information, which we have discussed in the previous section.

Considering the processing time and the "smooth" area in HiRISE that we are unable to fuse with a ground ORI, only specific areas are looked for in the NavCam to HiRISE coregistration. The rover positions are locked down for the areas where NavCam to HiRISE co-registrations are performed. Rover positions between any two co-registered areas are directly shifted after IBA or BA for Exterior Orientation (EO) correction. In this way, the global compliance guaranteed within the co-registered area, and the localisation accuracy between two co-registered areas are no longer a percentage of the distance from the landing site [12], but half of the distance between two co-registered areas. In the future, more sites of the three rover missions will be co-registered with HiRISE via the method described to improve the ground to orbit fusion accuracy.

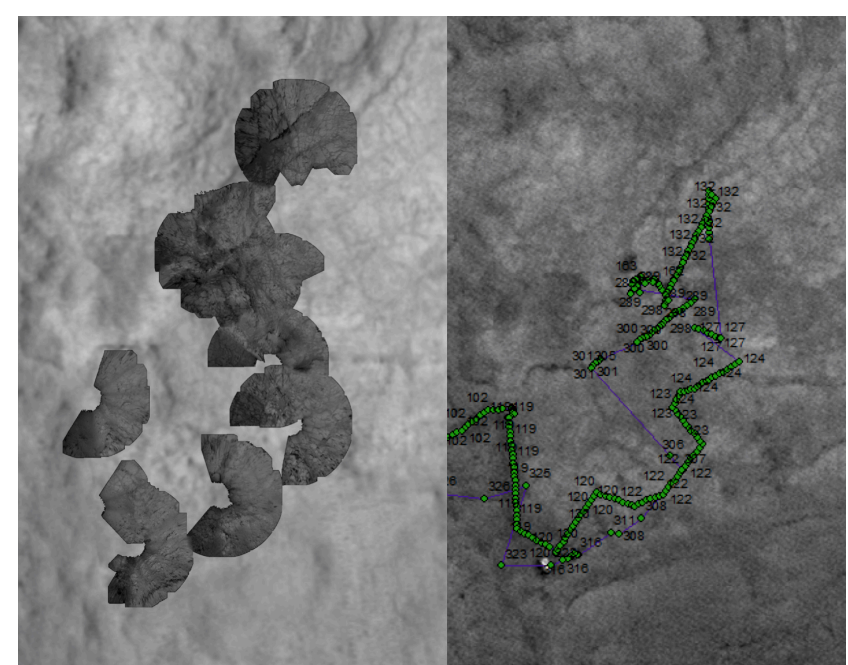

Figure 6 Example of co-registered wide baseline NavCam ORI mosaic and HiRISE ORI (left) and corrected traverse (right) for MSL from Sol 120 to Sol 179
In this work, we received the IBA corrected traverse for MERA and MER-B from OSU. The IBA corrected traverses were used as initial input in our localisation process. For MER-B, the NavCam to HiRISE co-registration was performed for 11 sites at Endurance Crater, 5 sites at Victoria Crater, and 2 sites at Santa-Maria Crater. For MER-A, the NavCam to HiRISE coregistration were performed for 2 sites with rover approaching Bonneville Crater and 5 sites at HomePlate. The IBA corrected traverses between co-registered areas are shifted, applying the same transformation of the localised positions.

For MSL, we use the traverse derived from a SPICE kernel as initial input. This is derived from the raw telemetry data. However, the HiRISE ORI is full of rich structural texture at site 5 Shaler, Yellowknife Bay, Gillespie Lake, and site 6 Cumberland up to the third PDS release on August 2013. The ground locations are then corrected using BA based on the coregistered positions at Site 5 and 6.

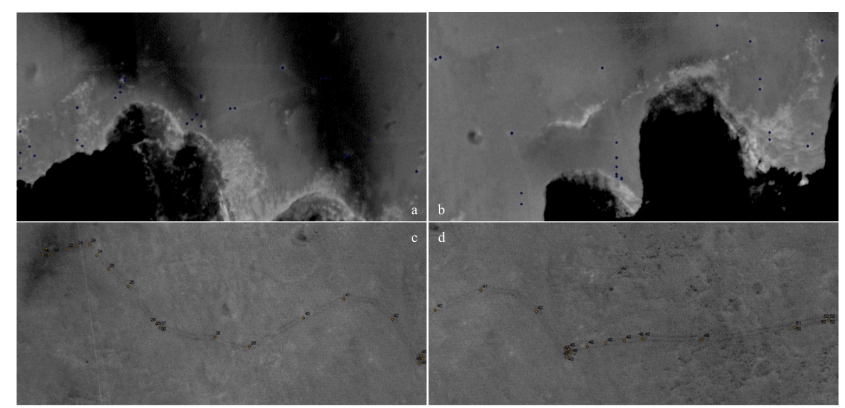

Figure 7. Co-registered rover positions on MER-B $(a, b)$ and MSL (c,d) HiRISE ORI showing alignment between the corrected traverse and rover tracks

In terms of validation, HiRISE images are captured at different times covering the same area for the three rover missions. These have been collected, projected, and co-registered with CTX and HRSC ORIs at MSSL. Some of them contain rover tracks in different areas. The corrected traverses are validated in the comparison of rover tracks appearing in different HiRISE images. For MER-A, there are not enough places containing good structural features that appear in both HiRISE and NavCam ORI mosaic for us to perform a co-registration. In such situations, we use an alternative workflow to derive the rover track segments in different HiRISE images, using the linefitting method to match the rover tracks with traverse segments after IBA correction. The traverse is validated using different rover track segments appearing in different HiRISE images.

\section{ASSESSMENT OF HIRISE-CTX-HRSC CO- REGISTRATION RESULTS}

In the HiRISE-to-CTX-to-HRSC co-registration stage, a quantitative evaluation of the automatically detected feature points (TPs) and manually selected control points (CPs) is undertaken for all three sets of maps. Table 1 shows the statistics of residuals of auto TPs and manually selected CPs for CTX to HRSC and HiRISE to CTX co-registration. Since we are using a global LSF adjustment method, which introduces a local transformation, the residuals of auto TPs are comparably low from 0.0056 pixels for MSL HiRISE-to-CTX coregistration to 0.0471 pixels for MER-B CTX-to-HRSC coregistration. Manual CPs are selected on viewable features, e.g. corners, line intersections, high local curvature points. They are relatively evenly distributed using a grid mode, i.e. 5-by-5 grid for MER-A and MSL, 8-by-8 grid for MER-B. It is impossible to select evenly distributed CPs in flat areas. However, in this way, evaluation on selected CPs will take into consideration the 
local distortion caused by LSF transformation. The average RMS errors of CTX-to-HRSC on manual CPs are relatively high mainly because there is a resolution gap of $6.5 \mathrm{~m} / \mathrm{pixel}$.

\begin{tabular}{|llllll|}
\hline & Methods & \multicolumn{2}{l}{ Auto TPs } & \multicolumn{2}{c|}{ Manual CPs } \\
\cline { 2 - 6 } MER- & RMSE & Avg. & Max & Avg. & Max \\
A & CTX-HRSC & 0.0145 & 0.0486 & 1.7697 & 2.6611 \\
& HiRISE- & 0.0073 & 0.0252 & 0.8695 & 1.2124 \\
MER- & CTX & & & & \\
B & CTX-HRSC & 0.0471 & 0.1734 & 2.7707 & 3.6165 \\
& HiRISE- & 0.0430 & 0.1390 & 0.3162 & 1.0382 \\
MSL & CTX & & & & \\
& CTX-HRSC & 0.0124 & 0.0319 & 0.9114 & 2.1344 \\
& HiRISE- & 0.0056 & 0.0093 & 0.6548 & 1.3201 \\
& CTX & & & & \\
\hline
\end{tabular}

Table 1 RMS Error from Auto TPs and Manual CPs At the final level, the ORI co-registration results were evaluated using the DTM in 10 test regions with different terrain types. We selected these test regions along or around the rover trajectory for each mission. Fig. 8 shows three selected elevation profiles from HRSC and HiRISE for MER-A.

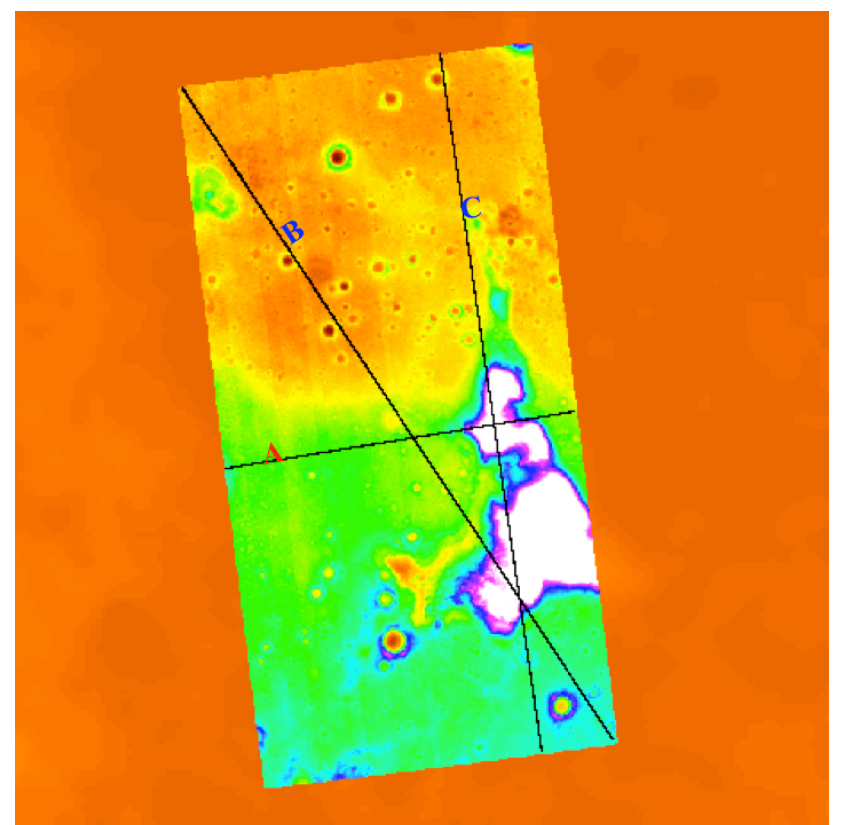

Figure 8 Example of co-registered MER-A HiRISE DTM on the top of HRSC DTM showing selected 3 profile lines A, B, and C Profile A

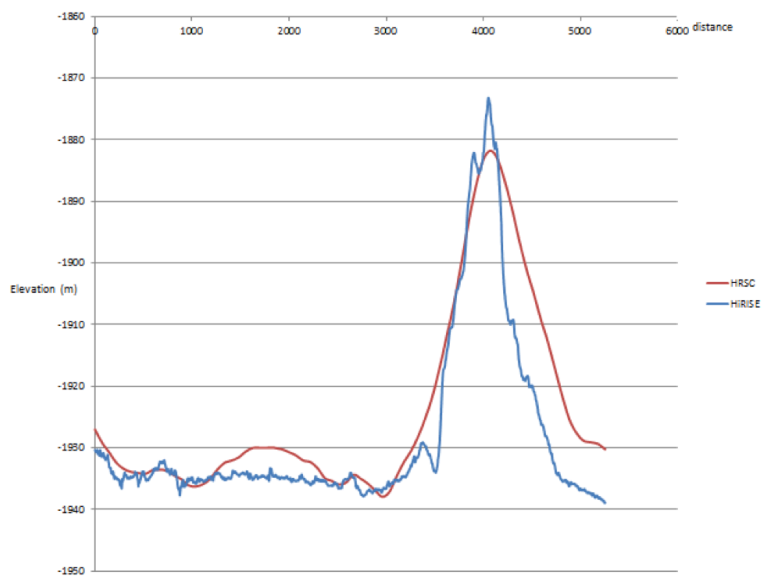

Figure 9. Profile A showing elevation of HiRISE (blue) and HRSC (red)

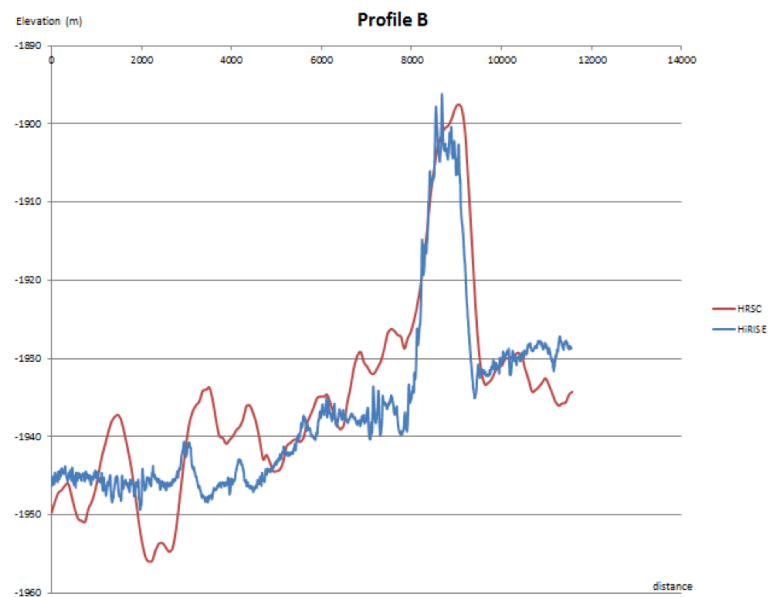

Figure 10. Profile B showing elevation of HiRISE (blue) and HRSC (red)

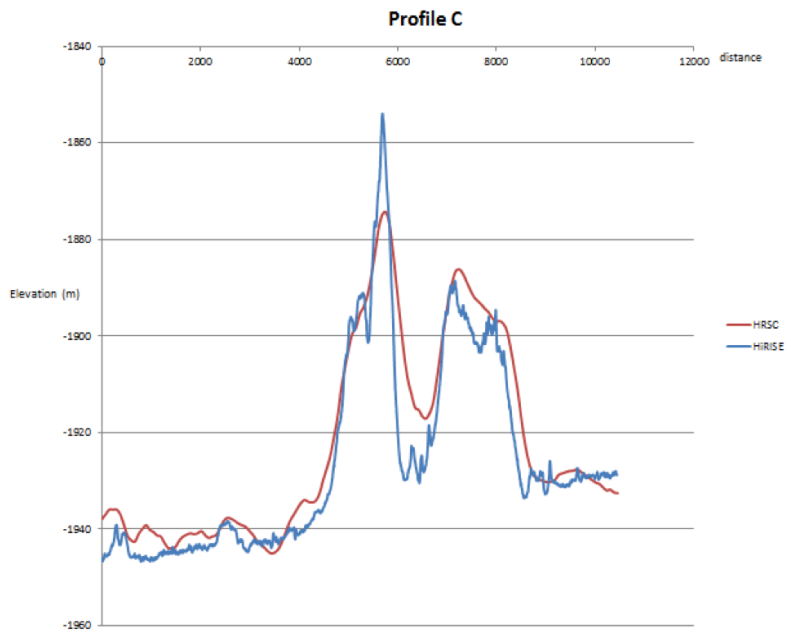

Figure 11. Profile $\mathrm{C}$ showing elevation of HiRISE (blue) and HRSC (red)

\section{APPLICATIONS}

\subsection{Visualisation of Multi-resolution 3D Products From Orbit to Ground Level}

The co-registered datasets and 3D products can be directly and interactively explored and scientifically analysed using the 3D viewer being developed by VRVis, a collaborator of the PRoViDE project. This tool applies advanced real-time rendering methods to enable smooth navigation through $3 \mathrm{D}$ reconstruction of planetary terrains. A high degree of realism is important to allow geological assessments. To achieve this goal, physical properties of rock material are considered for rendering. Different Bidirectional Reflectance Distribution Functions (BRDFs) are estimated from source images and implemented as "shaders" that directly run on a Graphical Processing Unit (GPU) and hence in real-time at MSSL. Besides changing material properties, natural illumination of the reconstructed scene is also important for a realistic impression. Skylight irradiance models for Mars and Earth will be applied for that purpose. A scientist can switch between both models and see how rock surfaces from Mars would appear on Earth enhancing the scientific analysis potential. These skylight models simulate not only the light from the sun for both planets but also the atmospheric scattering of light. The $3 \mathrm{D}$ viewer is a valuable additional tool for scientific analysis of planetary terrains and also serves as a planning tool for operations [13]. 


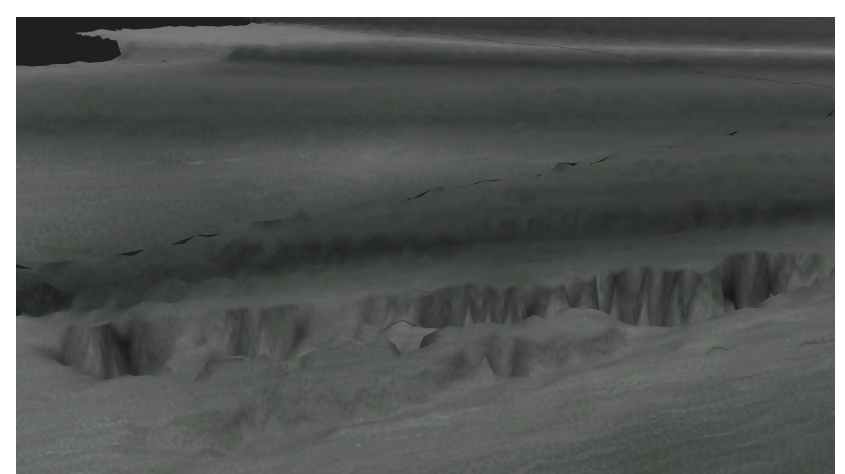

Figure 12. Example of real-time visualisation of reconstructed Martian terrain from MER images

\subsection{Interactive Web-GIS System For Science Target Selection}

Another important application of our localisation result is that the digitised multi ground level products can be accurately located in the global context, which will enable a "virtual geologist" to perform close-up visual analysis of key features (e.g. sedimentary layers) and make measurements (e.g. distance, dip and slope) with different levels of detail. Scientists are able to define geologies in global context and jump into a detailed local view of a 3D scene. Exemplarily the data is inserted into our interactive Web-GIS system, i.e. PRoGIS. The PRoGIS system [6] has been developed based on OGC protocols to provide visual and database search mechanisms for the display of stereo rover frames on a raster backdrop formed by coregistered multi-level orbital ORIs. The public will gain access to the Mars rover mission data (via UCL-MSSL's PDS mirror) and our seamlessly interconnected products within this unique geographical context. Additionally, PRoGIS also provides interactive photogrammetric operations, which are powered by the PRoViP (Planetary Robotics Vision Processing) system developed in PRoVisG, to initiate better understanding of the surface for expert users.

\section{CONCLUSIONS}

We have introduced our experimental method for producing multi-resolution co-registered $3 \mathrm{D}$ fused image products from orbit to ground level for MER-A, MER-B, and MSL. Technologies developed include the orbital datasets coregistration, wide baseline ground mapping, and ground to orbital co-registration. This paper has investigated the misregistration between publicly available HiRISE ORI/DTMs from the NASA HiRISE site and HRSC/MOLA with respect to the three rover missions, and introduced the unintended consequence that the rover traverse (and the associated SPICE kernel data for each and every rover image acquired in local coordinates) no longer match the landmarks observed in the HiRISE image after co-registration of HiRISE-CTX-HRSC. We have shown our automated processing chain for retrieving rover locations through co-registration from orbital to ground to bring everything into the same unique geo-referenced coordinate system with respect to HRSC, whose provenance is well documented with co-registration to MOLA [14]. Our multisensor co-registration based method guarantees a global compliance that does not have accumulated error in network based IBA localisation approaches in the ground to orbit fusion stage. The results are a set of co-registered ORI/DTMs from HiRISE, CTX, and HRSC, NavCam mosaics, and possible MSL-MastCam or MER-PanCam, etc. for the three rover missions, which can be interactively explored and analysed using our 3D viewer and web-GIS system. The developed processing chain could be further employed in future rover missions, e.g. ESA ExoMars 2018. Also in the future, further SPICE kernel updates will be derived to be consistent with all datasets from orbit to ground level.

\section{ACKNOWLEDGEMENT}

The research leading to these results has received funding from the European Community's Seventh Framework Programme (FP7/2007-2013) under grant agreement No.312377 PRoViDE.

\section{REFERENCES}

[1] G. Paar, J.-P. Muller, Y. Tao, T. Pajdla, J. Morley, K. Willner, I. Karatchevtseva, C. Traxler, G. Hesina, L. Tyler, D. P. Barnes, and S. Gupta, 2013, PRoViDE: Planetary Probes' Mass Vision Data Processing, European Planetary Science Congress (EPSC), London, UK, EPSC2013-289.

[2] W. Poole, and J.-P. Muller, 2013, Mars US rover traverse co-registration using multi-resolution Orbital 3D imaging datasets, EPSC 2013-661.

[3] M. J. Broxton and L. J. Edwards, 2008, The Ames Stereo Pipeline: Automated 3-D Surface reconstruction from Orbital Imagery, Proc. Lunar Planet, Sci., XXXIX, abstract 2419.

[4] J.-R. Kim and J.-P. Muller, 2009, Multi-resolution topographic data extraction from Martian stereo imagery, Planet Space Sci., 57: 2095-2112.

[5] D. Shin and J.-P. Muller, 2012, Progressively weighted affine adaptive correlation matching for quasi-dense $3 \mathrm{D}$ reconstruction. Pattern Recognition, 45(10): 3795 -3809.

[6] J. Morley, J. Sprinks, J.-P. Muller, Y. Tao, G. Paar, B. Huber, A. Bauer, K. Willner, C. Traxler, A. Garov, and I. Karachevtseva, 2014, Contextualising and Analysing Planetary Rover Image Products through the Web-Based PRoGIS, in EGU General Assembly, Vol. 16, EGU2014-16013.

[7] R. Li, et al, 2006, Spirit rover localization and topographic mapping at the landing site of Gusev crater, Mars, Journal of Geophysical Research, Vol. 111, E02S06.

[8] R. Li, J. Hwangbo, Y. Chen, and K. Di, 2011, Rigorous Photogrammetric Processing of HiRISE Stereo Imagery for Mars Topographic Mapping, IEEE Transactions on Geoscience and Remote Sensing, 49 (7): 2558-2572.

[9] R. L. Kirk, E. Howington-Kraus, M. R. Rosiek, J. A. Anderson, B. A. Archinal, K. J. Becker, D. A. Cook, D. M. Galuszka, P. E. Geissler, T. M. Hare, I. M. Holmberg, L. P. Keszthelyi, B. L. Redding, W. A. Delamere, D. Gallagher, J. D. Chapel, E. M. Eliason, R. King, A. S. McEwen, 2008, Ultrahigh resolution topographic mapping of Mars with MRO HiRISE stereo images: Meter-scale slopes of candidate Phoenix landing sites. J. Geophys. Res., 113 E00A24

[10] Y. Tao, and J.-P. Muller, 2013, A Multi-resolution 3D Reconstruction Tool: Exemplar using MSL NavCam PDS and MastCam PIO imagery, in EPSC (Europlanets), London, UK, EPSC2013-584.

[11] R. Li, S. He, Y. Chen, M. Tang, P. Tang, K. Di, L. Matthies, R. E. Arvidson, S. W. Squyres, L. S. Crumpler, T. Parker, and M. Sims, 2011, MER Spirit rover localization: Comparison of ground image- and orbital image-based methods and science applications, Journal of Geophysical Research, 116, E00F16.

[12] R. Li, K. Di, J. Wang, S. Agarwal, L. Matthies, A. Howards, and R. Willson, 2005, Incremental Bundle Adjustment Techniques Using Networked Overhead and Ground Imagery for Long-range Autonomous Mars Rover Localization, Proc. of The $8^{\text {th }}$ International Symposium on 
Artificial Intelligence, Robotics and Automation in Space ISAIRAS, ESA SP-603.

[13] C. Traxler, et al., 2014, An Interactive Virtual 3D Tool for Scientific Exploration of Planetary Surfaces, in EGU General Assembly, Vol. 16, EGU2014-12038.

[14] F. Scholten, et al., 2005, Mars Express HRSC data processing: Methods and operational aspects: Mapping Mars. Photogrammetric Engineering and Remote Sensing, 71:11431152. 